(2) OPEN ACCESS

${ }^{1}$ School of Medicine, Oregon Health \& Science University, Portland, Oregon, USA

2Department of Medicine, Oregon Health \& Science University, Portland, Oregon, USA

Correspondence to Dr André Martin Mansoor; mansooan@ohsu.edu

Accepted 19 September 2020

Check for updates

(c) BMJ Publishing Group Limited 2020. Re-use permitted under CC BY-NC. No commercial re-use. See rights and permissions. Published by BMJ.

To cite: Fillman $\mathrm{H}_{\text {, }}$ Riquelme P, Sullivan PD, et al. BMJ Case Rep

2020;13:e236437.

doi:10.1136/bcr-2020-

236437

\title{
Aseptic abscess syndrome
}

\author{
Hannah Fillman, ${ }^{1}$ Patricio Riquelme, ${ }^{2}$ Peter D Sullivan, ${ }^{2}$ André Martin Mansoor ${ }^{2}$
}

\section{SUMMARY}

A 43-year-old woman with Crohn's disease was admitted to the hospital with weight loss and 1 week of fever, abdominal pain and diarrhoea. At presentation, the patient was not on steroids or other immunosuppressive agents. Cross-sectional imaging of the abdomen revealed active colitis and multiple splenic and hepatic abscesses. All culture data were negative, including aspiration of purulent material from the spleen. Despite weeks of intravenous antibiotics, daily fever and abdominal pain persisted, the intra-abdominal abscesses grew, and she developed pleuritic chest pain and consolidations of the right lung. The patient was ultimately diagnosed with aseptic abscess syndrome, a rare sequelae of inflammatory bowel disease. All antimicrobials were discontinued and she was treated with high-dose intravenous steroids, resulting in rapid clinical improvement. She was transitioned to infliximab and azathioprine as an outpatient and repeat imaging demonstrated complete resolution of the deep abscesses that had involved her spleen, liver and lungs.

\section{BACKGROUND}

Aseptic abscess syndrome (AAS) is a rare but important clinical entity that affects patients with systemic inflammatory conditions, most often inflammatory bowel disease (IBD). Most patients are misdiagnosed with a bacterial infection and go on to receive prolonged courses of unnecessary antibiotics. Patients often undergo unnecessary surgical procedures (eg, splenectomy), which lead to substantial costs to their well-being and the healthcare system overall. This case highlights the importance of keeping the diagnostic door ajar, particularly when features of the case do not seem cohesive or when the clinical course does not go as expected.

There are 61 cases of AAS reported in the literature. ${ }^{1}$ Most patients respond favourably to highdose immunosuppression and achieve long-term remission with steroid-sparing agents. ${ }^{23}$ The key is establishing the diagnosis and starting immunosuppressive medications, which may initially appear to be a risky approach in a patient with the symptoms and signs of infection.

\section{CASE PRESENTATION}

A 43-year-old woman with Crohn's disease (CD) was admitted to the hospital with weight loss and 1 week of fever, abdominal pain and diarrhoea. As a result of medically refractory $\mathrm{CD}$, she underwent partial colectomy 1 year previously, and had been off immunosuppressive medications since that time. The patient was found to have multiple splenic and hepatic abscesses on cross-sectional imaging.
Treatment was initiated with broad-spectrum antibiotics, and she went on to receive various combinations of piperacillin-tazobactam, vancomycin, ceftriaxone, ampicillin-sulbactam, metronidazole and doxycycline. Despite nearly three continuous weeks of treatment, daily fever and abdominal pain persisted, and repeat imaging demonstrated interval growth of the intraabdominal abscesses. Moreover, the patient developed new pleuritic chest pain with rapidly enlarging consolidations of the right lung. She was then transferred to our institution for evaluation. On physical examination, the patient was febrile, tachycardic and tender to palpation throughout the upper abdomen. There was a pleural friction rub on auscultation of the base of the right lung as well as a two-component pericardial friction rub.

\section{INVESTIGATIONS}

On presentation, the peripheral white cell count was $14.5 \times 10^{9} / \mathrm{L}$ and serum $\mathrm{C}$ reactive protein was $294.1 \mathrm{mg} / \mathrm{L}$ (reference range $<10 \mathrm{mg} / \mathrm{L}$ ). Prior to transfer to our institution, serial cross-sectional imaging of the abdomen revealed active colitis and multiple splenic and hepatic abscesses that were growing in size (figure 1). Serial imaging of the chest demonstrated rapidly enlarging consolidations involving the right lung (figure 2). Several weeks after presentation, serum $\mathrm{C}$ reactive protein increased to $412 \mathrm{mg} / \mathrm{L}$. Multiple blood culture samples were negative. On two separate occasions, purulent material was aspirated from the spleen and tested using standard bacterial and fungal culture techniques as well as broad-range PCR (or 16S PCR), but no organisms were detected. The first aspiration was performed prior to the initiation of antibiotics. Serologies were negative for other organisms, including Mycobacterium tuberculosis, Brucella, Bartonella quintana, Bartonella henselae, Coxiella burnetii and Mycoplasma pneumoniae.

\section{DIFFERENTIAL DIAGNOSIS}

The initial working diagnosis in this case was intraabdominal infection related to active CD. However, the lack of clinical improvement despite several weeks of intravenous antibiotics did not support the theory of a typical bacterial infection. Moreover, the non-contiguous distribution of involvement (intraabdominal, lung, pericardium) raised suspicion for either a disseminating organism that was not being targeted by the antimicrobial regimen (eg, fungus) or a non-infectious process. Given the absence of an identified organism despite extensive testing, the diagnosis of AAS was considered. Review of the limited number of case reports indicated that the presentation of this case was highly consistent with AAS, including abdominal pain, weight loss, fever, deep sterile abscesses and the 

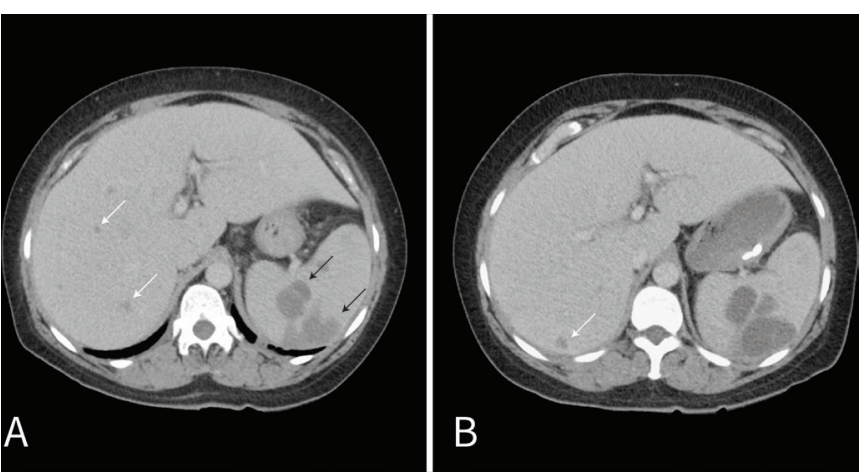

Figure 1 Serial cross-sectional imaging of the abdomen demonstrating multiple splenic (black arrows) and hepatic abscesses (white arrows) in a patient with Crohn's disease (A) that increased in size despite weeks of intravenous antibiotics (B).

lack of clinical improvement despite treatment with antibiotics in a patient with underlying IBD.

\section{TREATMENT}

After the diagnosis of AAS was made, all antimicrobials were discontinued and the patient was treated with high-dose intravenous steroids, resulting in rapid clinical improvement. She was transitioned to infliximab and azathioprine as an outpatient.

\section{OUTCOME AND FOLLOW-UP}

The patient continued infliximab and azathioprine as an outpatient with complete resolution of symptoms. Serum $\mathrm{C}$ reactive protein had decreased to $0.6 \mathrm{mg} / \mathrm{L}$. Repeat imaging 6 months after discharge from the hospital demonstrated total resolution of the deep abscesses that previously involved the spleen and liver (figure 3).

\section{DISCUSSION}

AAS is a rare manifestation of a variety of systemic inflammatory conditions, most often IBD. It is characterised by the formation of sterile collections of neutrophils in a variety of organs, including the spleen, skin, liver, lymph nodes and lungs. The literature concerning this entity is limited to case reports and case series. The initial case review published in 2007 on AAS reported 30 know cases in France and the Netherlands. ${ }^{3}$ A more recent literature review found 61 cases total. ${ }^{1}$ Only one other case has been reported in the USA. ${ }^{4}$

AAS can affect patients known to have CD or ulcerative colitis or may precede the diagnosis. The most common initial presentation is fever, abdominal pain, weight loss and peripheral

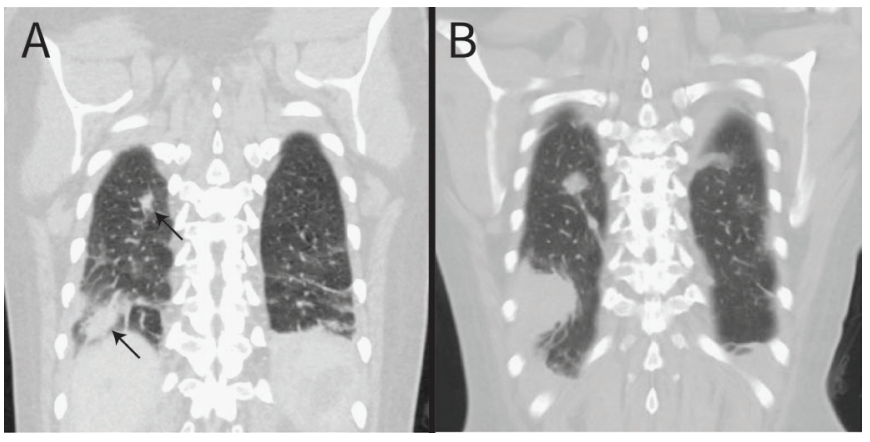

Figure 2 Serial cross-sectional imaging of the chest demonstrating right upper and lower lobe consolidations (arrows) in a patient with Crohn's disease $(A)$ that rapidly increased in size after a period of 10 days despite ongoing intravenous antibiotics (B).

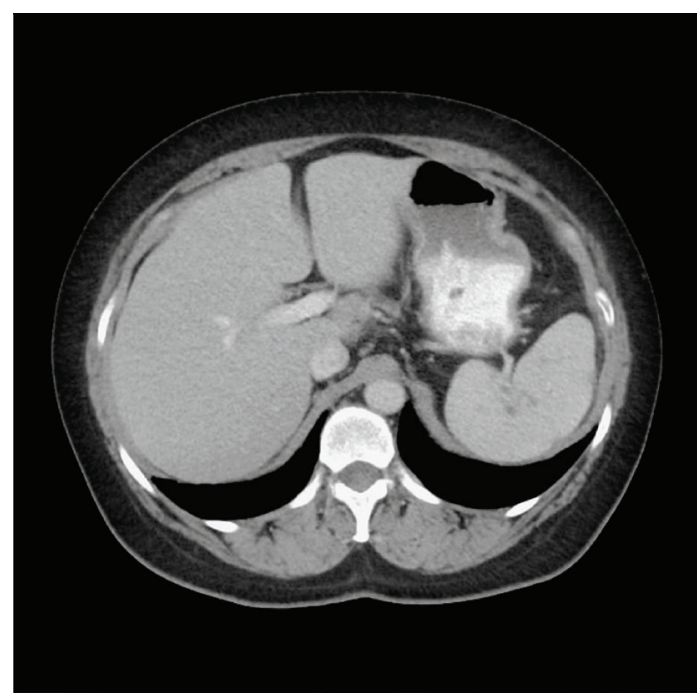

Figure 3 Complete resolution of splenic and hepatic abscesses in a patient with Crohn's disease after several months of treatment with immunosuppressive medications (compare to figure 1).

leucocytosis. Imaging shows deep abscess formation, most often involving the spleen and liver, but other potential sites of involvement include the lungs, lymph nodes, kidneys, pancreas, testes and brain. It is important to consider colonoscopy when previously healthy patients are diagnosed with AAS given that it can be the initial manifestation of IBD. ${ }^{235}$ The pathophysiology of AAS is not clearly understood. The characteristic pathological finding of sterile neutrophilic infiltration of the deep tissues is similar to that of the neutrophilic dermatoses, which is characterised by sterile neutrophilic infiltration of the skin. Pyoderma gangrenosum (PG) is an example of a neutrophilic dermatosis that is often associated with IBD. Whether AAS and PG belong to a spectrum of the same underlying autoinflammatory condition is not clear. However, the histopathology between the two appears to be different: the deep abscesses of AAS are surrounded by a granulomatous reaction, which is absent in the classical form of PG. ${ }^{3}$

There are no formal diagnostic criteria for AAS. Ultimately, AAS is a diagnosis of exclusion in a person with radiological evidence of deep abscesses in the absence of infection. In our case, infection was ruled out by all reasonable means prior to the initiation of steroids. This included bacterial and fungal blood cultures, serologic testing for viral and bacterial aetiologies, and sampling splenic abscesses on two occasions, with purulent material tested with culture and PCR.

Antibiotic therapy is universally ineffective for AAS. However, the vast majority of patients $(\sim 95 \%)$ respond to corticosteroids. ${ }^{2}$ Initial steroid treatment ranges from 0.5 to $1 \mathrm{mg} / \mathrm{kg} /$ day with a taper starting after $2-4$ weeks. $^{6}$

The ideal regimen for maintenance therapy is still evolving. In patients placed on steroid maintenance therapy alone relapse occurred in some, but not all patients. ${ }^{2}$ Disease-modifying antirheumatic drugs (DMARDs) and biological targeted agents have been used as maintenance therapy. DMARDs that have been used to treat AAS include azathioprine, colchicine, cyclophosphamide, ciclosporin, methotrexate and mycophenolate mofetil. Biological targeted therapies that have been used include infliximab, adalimumab, etanercept, anakinra, canakinumab and tocilizumab. ${ }^{16}$

Long term, roughly two-thirds of the documented patients had disease relapse. Relapse can occur as early as 2-4 weeks 
Patient's perspective

A 5-hour ambulance ride later, I was put into a room with two other patients at OHSU. I was quickly assigned several teams of doctors from different specialties and my parents and I spent our first 24 hours meeting them all and getting an idea of the strategy going forward. The specialties were General Medicine (primary team), Gastroenterology, Infectious Diseases, and Pulmonology. There were 3-5 people in each of the teams and at least one representative from each team visited me pretty much every day.

Initially at OHSU, I was kept on IV antibiotics and pain medicine. More scans were done of my abdomen and lungs and the abscesses appeared to be continually growing and had spread to my lungs. The culture results kept coming in completely negative for anything bacterial or even fungal. The scans also showed inflammation in my large bowel, which gastroenterology thought was probably Crohn's disease related. The treatment of Crohn's disease is usually steroids and immunosuppressants. However, with unknown abscesses on board, no one wanted to suppress my immune system.

Then a new idea was brought to the table: Aseptic Abscess Syndrome (AAS). The lead doctor on my general medicine team recalled a case of AAS that his colleague had seen years before, and felt that my symptoms seemed to match his recollection. I was soon given three articles written in Canada and France which described AAS, and it really made sense to me. Most of the known patients (less than 100 documented in the world) had an inflammatory bowel disease, and all of them had found antibiotics completely useless in the treatment of their abscesses. The only effective initial treatment was STEROIDS.

Thus began the arguments between my teams of doctors. To be fair, none of them had ever seen a case of AAS before and the only articles on it were rather brief case studies from outside the United States. The infectious diseases doctors had never seen abscesses that were not the result of some sort of infection. They were extremely nervous about the idea of stopping the antibiotics and switching to steroids. If I had an infection, the steroids would make it worsen very rapidly.

The group of general medicine doctors seemed to be the only ones who wanted to try switching to steroids. I also was leaning in this direction after talking with my parents and reading the articles on AAS. It just made the most sense to me based on my symptoms and the ineffectiveness of antibiotics and the lack of any growth on cultures. When I told the other groups of doctors that I wanted to stop antibiotics, they really gave me a hard time. At one point someone told me to not listen to anything the general medicine people said. It was extremely frustrating.

Finally, we just did the switch though. And, lo and behold, things began to improve! I was on high doses of steroids and no antibiotics at all. It took about 10 days of this and I was well enough to go home. By the end of my stay at OHSU, my liver abscesses were almost completely gone, the spleen abscesses were significantly fewer in number and smaller, and my lung abscesses had also shrunk. I no longer had fevers and my pain was manageable.

After I was discharged, I remained on steroids before transitioning to the combination of infliximab and azathioprine. It is now more than 16 months later and I have not had any resurfacing of the AAS symptoms.

after initial treatment or as late as 7 years. ${ }^{6}$ Importantly, the recrudescence of abscesses in these patients should prompt a comprehensive reevaluation as ongoing immunosuppressive therapy puts them at increased risk for opportunistic infections,
Learning points

- Aseptic abscess syndrome can occur in patients with inflammatory bowel disease and is often misdiagnosed as infection. Failure to recognise this entity can lead to unnecessary morbidity and healthcare costs.

- Aseptic abscess syndrome must be considered when patients with inflammatory bowel disease develop deep abscesses that do not respond to conventional antibiotic therapy.

- The initial treatment of choice for aseptic abscess syndrome is high-dose intravenous glucocorticoids. Maintenance therapy should include a disease-modifying antirheumatic drug or biologically targeted agent or a combination of the two.

including those that can be associated with abscess formation, such as tuberculosis. ${ }^{7}$ Our patient was maintained on both infliximab and azathioprine and has not experienced a relapse after a year and a half of treatment. Further research into combination therapy with a DMARD and biological agent would be helpful, although challenging to execute given the small number of cases of AAS worldwide.

Overall survival in those diagnosed with AAS is favourable. There have been no reported fatalities directly linked to AAS. However, morbidity can be considerable, particularly in undiagnosed cases.

Twitter André Martin Mansoor @AndreMansoor

Acknowledgements AMM thanks Christopher Kwock for serving as an unofficial consultant during the care of the patient. The authors thank Joseph Nugent for polishing the figures used in this case.

Contributors HF, PR and AMM were directly involved in the care of the patient. AMM made the diagnosis. PDS served an unofficial consulting role during the care of the patient. AMM planned and coordinated the creation of the case report. HF, PR and AMM created the figures. HF and AMM primarily wrote the manuscript. PR, PDS and AMM edited and refined the manuscript.

Funding The authors have not declared a specific grant for this research from any funding agency in the public, commercial or not-for-profit sectors.

Competing interests None declared.

Patient consent for publication Obtained

Provenance and peer review Not commissioned; externally peer reviewed.

Open access This is an open access article distributed in accordance with the Creative Commons Attribution Non Commercial (CC BY-NC 4.0) license, which permits others to distribute, remix, adapt, build upon this work non-commercially, and license their derivative works on different terms, provided the original work is properly cited and the use is non-commercial. See: http://creativecommons.org/ licenses/by-nc/4.0/.

\section{REFERENCES}

1 Elessa D, Thietart S, Corpechot C, et al. TNF- $\alpha$ antagonist infliximab for aseptic abscess syndrome. Presse Med 2019;48:1579-80.

2 Bollegala N, Khan R, Scaffidi MA, et al. Aseptic abscesses and inflammatory bowel disease: two cases and review of literature. Can J Gastroenterol Hepatol 2017; 2017:1-8

3 André MFJ, Piette J-C, Kémény J-L, et al. Aseptic abscesses: a study of 30 patients with or without inflammatory bowel disease and review of the literature. Medicine 2007:86:145-61.

4 Doll R, Kenneth $F$, Hostoffer $R$, et al. A case of prolonged remission following splenectomy. Am J Gastroenterol 2018;113:1264-5.

5 Bavaro DF, Ingravallo G, et. al. Splenic abscesses as a first manifestation of Crohn's disease: a case report. 2019;19:144

6 André M, Aumaître 0. [Aseptic abscesses syndrome]. Rev Med Interne 2011;32:678-88.

7 Bavaro DF, Fiordelisi D, Angarano G, et al. Targeted therapies for autoimmune/idiopathic nonmalignant diseases: risk and management of opportunistic infections. Expert Opin Drug Saf 2020;19:817-42. 
Copyright 2020 BMJ Publishing Group. All rights reserved. For permission to reuse any of this content visit https://www.bmj.com/company/products-services/rights-and-licensing/permissions/

BMJ Case Report Fellows may re-use this article for personal use and teaching without any further permission.

Become a Fellow of BMJ Case Reports today and you can:

- Submit as many cases as you like

- Enjoy fast sympathetic peer review and rapid publication of accepted articles

Access all the published articles

- Re-use any of the published material for personal use and teaching without further permission

Customer Service

If you have any further queries about your subscription, please contact our customer services team on +44 (0) 2071111105 or via email at support@bmj.com.

Visit casereports.bmj.com for more articles like this and to become a Fellow 